\title{
The Effect of Smaller Turbulent Motions on Heat Transfer in the
}

\section{Annular Gap Flow of Flywheel}

\author{
Y.J. Wang, D.Z. Wang, W.Z. Guo, J.L. Yin, Y.Y. Hu
}

School of Mechanical Engineering, Shanghai Jiao Tong University, Shanghai, People's Republic

of China

ABSTRACT: The forced convective heat transfer in the gap of a flywheel is affects the safety of reactor coolant pump (RCP), such as the operation temperature limit of the bearing, therefore it is necessary to analyze the heat transfer in the annular gap flow of a flywheel. The current study provides evidence that the convective heat transfer depends on the flow structure. When the Taylor number of this flow is about 1E12, the flow structure has a special pattern likes Herringbone near the wall, and consists of many smaller Görtler vortices. Although the earliest experimental studies found the Görtler vortex with laser-induced fluorescence (LIF), these vortices are difficult to study by numerical analysis with direct numerical simulation (DNS) or the large eddy simulation (LES), and the Reynolds-averaged Navier-Stocks (RANS) cannot capture the smaller scale vortices in the wall boundary layers with overestimated eddy viscosity. In this paper, we adopt the Partially-averaged Navier-Stokes (PANS) model to observe the smaller turbulent motions in the annular gap flow of a flywheel, and analyze the effect of Görtler vortices on convective heat transfer. Compared with $k-\varepsilon$, PANS simulation suggests that there are larger temperature gradients at the Görtler vortices region, and two temperature mixing processes occur at the boundaries of a pair of Taylor vortices.

Keywords: Flywheel, Taylor-Couette flow, Heat transfer, PANS, Görtler vortices.

\section{Nomenclature}

\begin{tabular}{|c|c|c|c|}
\hline$\mu$ & dynamic viscosity, $\mathrm{N} \cdot \mathrm{m}^{2} / \mathrm{s}$ & $x_{j}$ & Cartesian coordinate, $j=1,2,3$ \\
\hline$\mu_{t}$ & turbulent eddy viscosity & $\Delta$ & the smallest grid dimension \\
\hline$N$ & kinematic viscosity, $\mathrm{m}^{2} / \mathrm{s}$ & $\Lambda$ & the turbulent length scale \\
\hline$T$ & time,s & $R e_{i}$ & shear Renolds number \\
\hline$\omega$ & rotation velocity, $\mathrm{rad} / \mathrm{s}$ & & \\
\hline$\sigma_{\varepsilon}$ & turbulent dissipation Prandtl number & & \\
\hline$\sigma_{k}$ & turbulent kinetic energy Prantl number & & \\
\hline$\varepsilon$ & turbulent kinetic dissipation & & \\
\hline$\varepsilon_{u}$ & unresolved dissipation & & \\
\hline$C_{\mu}$ & $k-\varepsilon$ turbulent model constant & & \\
\hline$D_{h}$ & hydraulic diameter, $\mathrm{m}$ & & \\
\hline$f_{r}$ & curvature correction function & & \\
\hline$f_{\varepsilon}$ & unresolved-to-total ratios of kinetic dissipation & & \\
\hline$f_{k}$ & unresolved-to-total ratios of kinetic energy & & \\
\hline $\boldsymbol{k}$ & turbulent kinetic energy & & \\
\hline $\boldsymbol{k}_{\boldsymbol{u}}$ & unresolved turbulent kinetic energy & & \\
\hline $\mathrm{Nu}$ & Nusselt number & & \\
\hline Ta & Taylor number, $T a=\frac{R_{o} \omega^{2} D_{h}^{2}}{v^{2}}$ & & \\
\hline
\end{tabular}

Corresponding author:+86 1381800036;

E-mail: dzwang@sjtu.edu.cn (D.Z. Wang) 


\section{Introduction}

In an advanced reactor coolant pump, the gap flow between flywheel and stationary shell is directly linked to the security of pump operation, such as the friction loss of the flywheel and the operation temperature of upper bearing. Once the design is not rational, the heat cannot be transferred from the pump in a short time, the risk of accident of pump will increase. For the security of pump, a closed chamber flow is considered in the upper flywheel of RCP in order to increase the efficiency of heat transfer. Fig. 1 presents this simplified configuration of the closed chamber flow, the primary internal water flow from upper radial bearing into the cavity, and then flow from the bottom outlet to the heat exchanger, thus the primary internal water will not flow upward, so this closed chamber flow without axial flow is called Taylor-Couette flow.

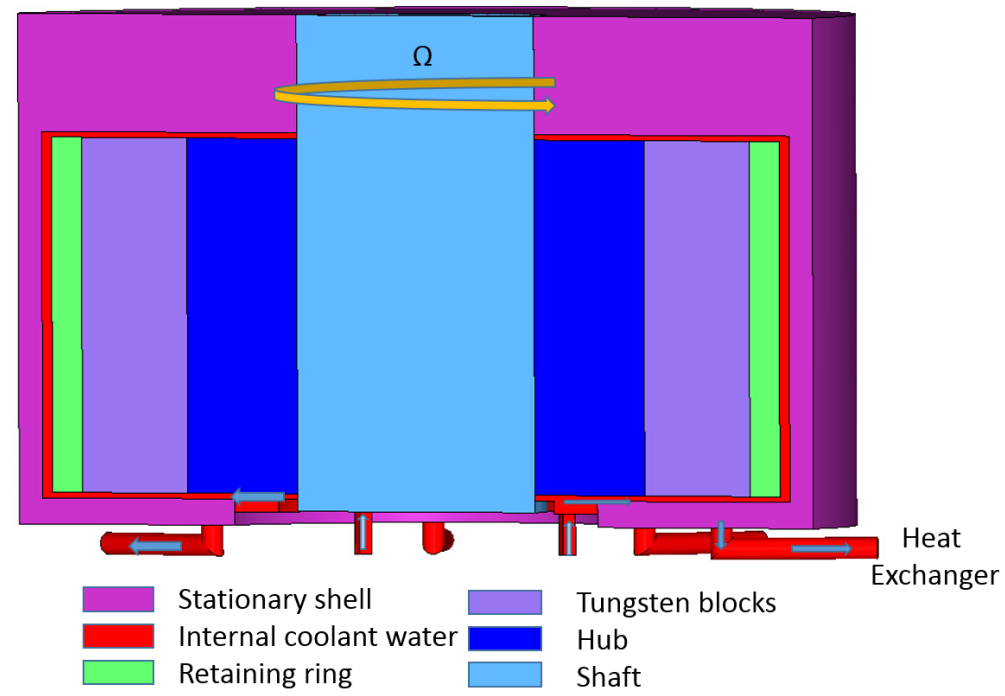

Fig. 1 Configuration of flywheel

Taylor-Couette flow has been investigated extensively since the earliest studies by Mallock and Taylor (Mallock, 1896; Taylor, 1923), most studies focused on the primary instability and the flow state regimes, the flow state regimes had been classified according to the different flow state (Andereck et al., 1986; van Gils et al., 2011). For a high Renolds number of the Taylor-Couette flow $\left(R e_{i}>1 \mathrm{E} 6\right)$, the flow is classified as turbulent Taylor Vortex Flow (TTVF), it is a more complex flow state. Barcilon (Barcilon et al., 1979) and co-workers first discovered a remarkable smaller scale Görtler vortex near the surface of the rotating cylinder and the stationary cylinder at moderately high Renolds number, corresponding to the thickness of the boundary layer, the organized flow pattern is described as 'Herringbone', Fig. 2 shows the schematic of the this special pattern (Barcilon and Brindley, 1984). Wei firstly investigated the Görtler vortices in the Taylor-Couette flow with the laser-induced fluorescence (LIF), the experiment indicates the Görtler vortices form at the inner rotating cylinder and become stronger with the increasing of the Renolds number, the strength of the Görtler vortex mechanism is governed by the radius of the curvature and the gradient of the velocity (Wei et al., 1992). Recent years, with the help of the high-performance computer, the full 3D simulation of the turbulent Taylor-Couette has been widely studied. Batten adopted direct numerical simulation (DNS) to study Turbulent Taylor-Couette flow, but Renolds numbers of his study are 5E3 and 8E3, the cost of the DNS were beyond his available computing resources, so he performed a $2 \mathrm{D}$ simulation rather than a full 3D simulation (Batten et al., 2002). Dong performed 3D simulations at four Renolds numbers 
( $R e=1 \mathrm{E} 3 \sim 8 \mathrm{E} 3)$, he discovered that Görtler vortices are stretched azimuthally at high rotational speed, and extend away from the wall to the core of the gap. The angle between the stretch direction and the boundary of Taylor vortices is called tilting angle, and he analyzed the range of the tilting angle with Barcilon's theory analysis (Dong, 2008a; Dong, 2008b). Bilson also used DNS to perform the same magnitude order of the Renolds number as Dong ( $R e=3.2 \mathrm{E} 3)$, his study suggests the Görtler vortices will increase the azimuthal wall shear at the 'Outflow' and 'Inflow' boundary of two Taylor vortices (Bilson and Bremhorst, 2007). Brauckmann performed a DNS simulation of the Taylor-Couette flow ( $T a \approx 3 \mathrm{E} 4$ ), one objective of his study is to evaluate the relation between torque and boundary layer thickness, the angular velocity profiles exhibit different due to the small scale turbulent motions, and the torque will changed as the angular moment (Brauckmann and Eckhardt, 2013). Chouippe and Climent used DNS to observe the transition of the flow states $(R e=3 \mathrm{E} 3 \sim 8 \mathrm{E} 3)$, and found the migration bubbles accumulated in 'Herring-bone' near the inner cylinder (Chouippe et al., 2014).

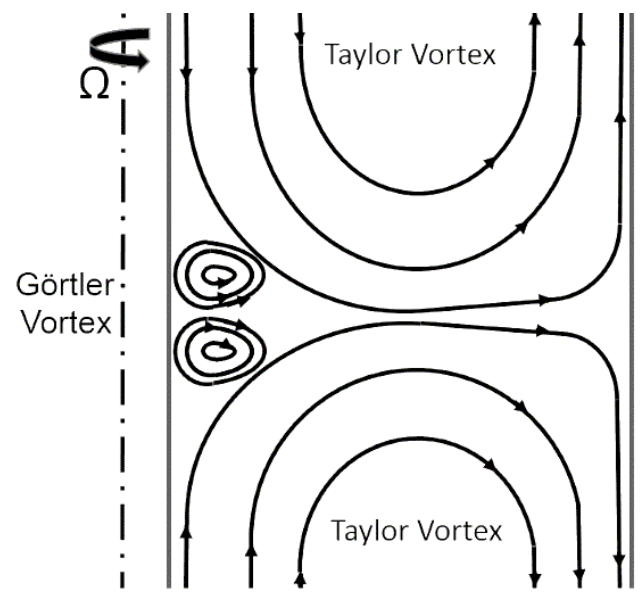

Fig. 2 Flow state of the annular gap flow

Heat transfer in gap flow of the Taylor-Couette flow was studied by experiments (Aoki et al., 1967; Becker and Kaye, 1962; Tachibana and Fukui, 1964; Tzeng, 2006). Their researches based on their own experimental configuration indicate Nusselt number varies with the Taylor number. In 2011, Fènot investigated these previous work and plotted these experimental results in the same figure, and then formulated the experimental correlation of the Nusselt number and Taylor number, $N u=A \cdot T a^{n}, A$ and $n$ are the constants depending on the experiments (Fénot et al., 2011). In Fènot's review, Tachibana and Fukui are the only authors conducting an experiment at a high Taylor number closed to the annular gap flow of the flywheel ( $T a=1 \mathrm{E} 12)$, but the gap-to-radius ratio is . Besides that, most of researches just considered the global heat transfer, the effect of the Görtler vortices on local heat transfer near the wall is still not studied extensively, it is reported that Gayoub is the only one who analyzed the heat transfer of the Taylor-Couette at $T a=2 \mathrm{E} 6$, Ghayoub's experiment suggests that Nusselt number reaches a peak value at the boundaries of the Taylor vortices (Fénot et al., 2011). In short, it is still not clear that the effect of the small scale of the turbulent motion on the heat transfer for the higher Reynolds number $(R e>3 \mathrm{E} 4)$.

Compelled by the strict security requirements of the nuclear power plant, it is necessary to adopt a proper numerical method to predict the effect of the small scale of turbulent motions on the temperature distribution. However, an accurate numerical method of the heat transfer depends on the eddy-resolving method for turbulent flows (Fröhlich and von Terzi, 2008). As is mentioned above, the DNS costs larger computational resources, and the RANS are not able to provide 
accurate and detailed information regards to the unsteady flow. For realizing the smooth transition from DNS to RANS, Girimaji et al. developed partially averaged Navier-Stocks (PANS) method in order to obtain different physical resolution. The physical resolution of PANS method depends on the unresolved-to-total ratios of turbulent kinetic energy $f_{k}$ and dissipation $f_{\varepsilon}$ (Girimaji et al., 2003). By specifying these control parameters, the effective viscosity is changed, leading to the liberation of smaller scale turbulent motions. PANS method has been used to analyze the structure of unsteady turbulent flow, such as backward-step flow, Karman vortex street. For the high Renolds number simulation, these studies indicate that $f_{k}$ is the key parameter for the small scale motion (Lakshmipathy and Girimaji, 2010). More detailed information about PANS method can be found in Girimaji's papers (Girimaji, 2006; Girimaji and Abdol-Hamid, 2005). In summary, PANS method can be utilized for high Renolds number flow with less computational cost.

The objective of this paper is to analyze the effect of Görtler vortices on the heat transfer in annular gap flow of flywheel with PANS method. A two-equation PANS model based on the standard $k-\varepsilon$ model is presented. Moreover, by comparing with the data of Tachibana's experiments, a proper value of $f_{k}$ is determined for the heat transfer in the annular gap flow. Furthermore, we used the PANS model to analyze the annular gap flow of the flywheel, and analyzed the effect of the Görtler vortices on the heat transfer. Finally, the findings and conclusions of the paper are summarized.

\section{PANS Model Formulation}

PANS model has been proposed aiming to compute the unsteady turbulent flows, more scales of turbulent motion will be resolved than RANS depending on the values of the filter control parameters (Girimaji, 2006),

$$
f_{k}=\frac{k_{u}}{k} ; \quad f_{\varepsilon}=\frac{\varepsilon_{u}}{\varepsilon}
$$

The PANS presented in this paper is derived from the standard $k-\varepsilon$ model, it has the same form of closed equations.

$$
\begin{gathered}
v_{t}=C_{\mu} \frac{k_{u}^{2}}{\varepsilon_{u}} \\
\frac{\partial k_{u}}{\partial t}+U_{j} \frac{\partial k_{u}}{\partial x_{j}}=P_{u} f_{r}-\varepsilon_{u}+\frac{\partial}{\partial x_{j}}\left(\left(v+\frac{v_{t}}{\sigma_{k u}}\right) \frac{\partial k_{u}}{\partial x_{j}}\right) \\
\frac{\partial \varepsilon_{u}}{\partial t}+U_{j} \frac{\partial \varepsilon_{u}}{\partial x_{j}}=C_{e 1} f_{k} \frac{P_{u} f_{r} \varepsilon_{u}}{k_{u}}-C_{e 2}^{*} \frac{\varepsilon_{u}^{2}}{k_{u}}+\frac{\partial}{\partial x_{j}}\left(\left(v+\frac{v_{t}}{\sigma_{\varepsilon u}}\right) \frac{\partial \varepsilon_{u}}{\partial x_{j}}\right)
\end{gathered}
$$

where

$$
C_{e 2}^{*}=C_{e 1}+\frac{f_{k}}{f_{e}}\left(C_{e 2}-C_{e 1}\right) ; \quad \sigma_{k u}=\sigma_{k} \frac{f_{k}^{2}}{f_{\varepsilon}} ; \quad \sigma_{\varepsilon u}=\sigma_{\varepsilon} \frac{f_{k}^{2}}{f_{\varepsilon}} ;
$$

The subscript $u$ indicates unresolved statistics in PANS model, $K_{u}$ and $\varepsilon_{u}$ are the unresolved kinetic energy and dissipation respectively, and unresolved-to-total ratios of kinetic energy $f_{k}$ and dissipation $f_{\varepsilon}$ are the two control parameters, $f_{k}$ and $f_{\varepsilon}$ determine the turbulent length scale. By varying $f_{k}$ and $f_{\varepsilon}$, the filter width of the energy spectrum can be controlled. If $f_{k}=f_{\varepsilon}=1$, PANS 
model will degenerate to RANS. On the contrary, with the decrease of $f_{k}$, PANS model will become DNS (Girimaji, 2006; Girimaji et al., 2003). In the early stage, the value of $f_{k}$ was defined as a constant, so the early PANS is also called a fix-point analysis. Actually, the resolved turbulence length scale is related to the local dissipation and local eddy viscosity in Kolmogorov's universal equilibrium theory, so the turbulence length scale is not unit in the resolved domain, the turbulent viscosity is dependent on the $f_{k}$, so Girmaji proposed a formula for $f_{k}$,

$$
f_{k}=\frac{1}{\sqrt{C_{\mu}}}\left(\frac{\Delta}{\Lambda}\right)^{\frac{2}{3}}
$$

where $\Delta$ is the smallest grid dimension, and $\Lambda$ is the turbulent length scale, and the turbulent viscosity coefficient $C_{\mu}$ is prescribed as the same value in RANS model. Although the formula of $f_{k}$ proposed by Girimaji is a great progress for PANS model, sometimes the value of $f_{k}$ is larger than 1 , which will cause computation errors. Therefore, there is still no better way to determine the value of $f_{k}$.

In addition, for the standard $k-\varepsilon$ model, a disadvantage of the eddy-viscosity models is insensitive to streamline curvature and system rotation, so the production term is not estimated properly. For a more accurate simulation, a curvature correction function $f_{r}$ related to the strain rate and vorticity tensor is present(Spalart and Shur, 1997), this curvature correction function is also used in the PANS model to overcome the drawback inherited from the standard $k-\varepsilon$ model.

Besides that, total energy equation is adopted for the heat transfer model, and the viscosity heating has been considered in the total energy equation.

In this study, the commercial CFD code CFX is used, the unresolved scale turbulent motions depend on the PANS simulations with a constant value of $f_{k}$.

\section{Experimental comparison}

\subsection{Tachibana's experiment}

A heat transfer experiment of the Taylor-Couette flow Tachibana conducted are used to be a benchmark to verify the validity of the PANS model, because it has the same order of magnitude as the Taylor number of the gap flow of flywheel, and water is used in the experiment, the material property is variation with temperature. Fig. 3 shows the configuration of Tachibana's experimental apparatus.

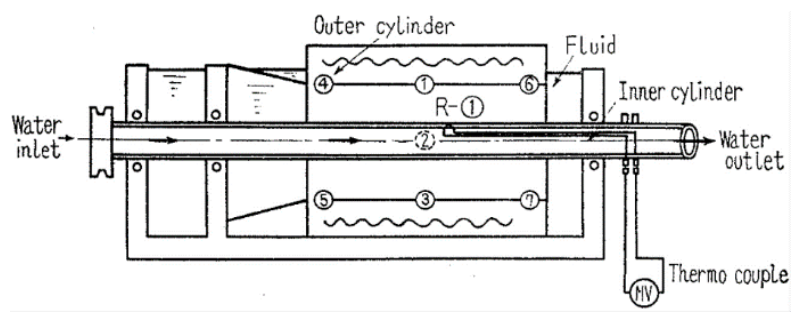

Fig. 3 Tachibana experimental apparatus(Tachibana and Fukui, 1964)

The inner and outer radii of the annular gap are $0.015 \mathrm{~m}$ and $0.068 \mathrm{~m}$, respectively, and the length of the test section is $0.25 \mathrm{~m}$, the rotational speed is from $228 \mathrm{rpm}$ to $2700 \mathrm{rpm}$. Moreover, the outer surfaces of the stationary cylinder and shaft are wound with nichrome wire for heating, and the inner cylinder is a hollow shaft, the water flows through the inside of the shaft to control the temperature of the shaft. In addition, two different heating methods are used in the experiments, one is the heated stationary cylinder and cooled rotor, another one is the heated inner cylinder and cooled stationary cylinder. Although the heating methods influence the temperature 
distribution, the experimental results suggest that the two different heat transfer forms have the same heat transfer mechanism (Tachibana and Fukui, 1964). Therefore, the average Nusselt number of his experiments gave the flowing empirical equation:

$$
N u=0.046(T a \cdot \operatorname{Pr})^{1 / 3}, 1 E 8 \leq T a \leq 2.5 E 13
$$

\subsection{PANS simulation}

A similar numerical model is set up to compare the result of Tachibana's experiments, as is shown in Fig. 4. In this model, the temperature of the outer surface of the stationary cylinder is set to be constants $\left(T_{\text {inner }}=283.15 \mathrm{~K}, T_{\text {outer }}=323.15 \mathrm{~K}\right.$ ), the two ends of the model are considered as adiabatic wall. The grid size of the numerical model is $240 \times 40 \times 100$ (hoop $\times$ radial $\times$ length), the number of grid is 960000 . The model is used to simulate the flow state at five different rotational speed cases (500 rpm, $1000 \mathrm{rpm}, 1500 \mathrm{rpm}, 2000 \mathrm{rpm}, 2700 \mathrm{rpm}$ ),

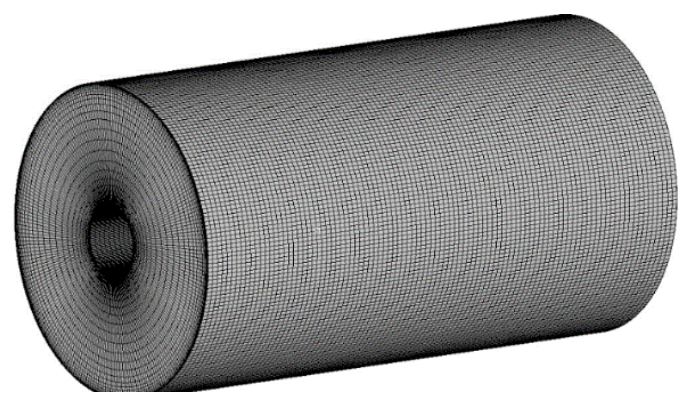

Fig. 4 Computational mesh for the Tachibana's experiment

In addition, for analyzing the influence of $f_{k}$ on the heat transfer, $f_{k}$ is specified as 1 , $0.7,0.5,0.3$ and 0.1 . By comparing the flow state, we find that with decreasing $f_{k}$, the PANS model resolved the small scale vortices, and these unsteady small scales vortices appear close to the inner rotating cylinder wall, according to the research of Fénot, the small scale vortices can influence the local heat transfer. The difference is shown in the Fig. 5.

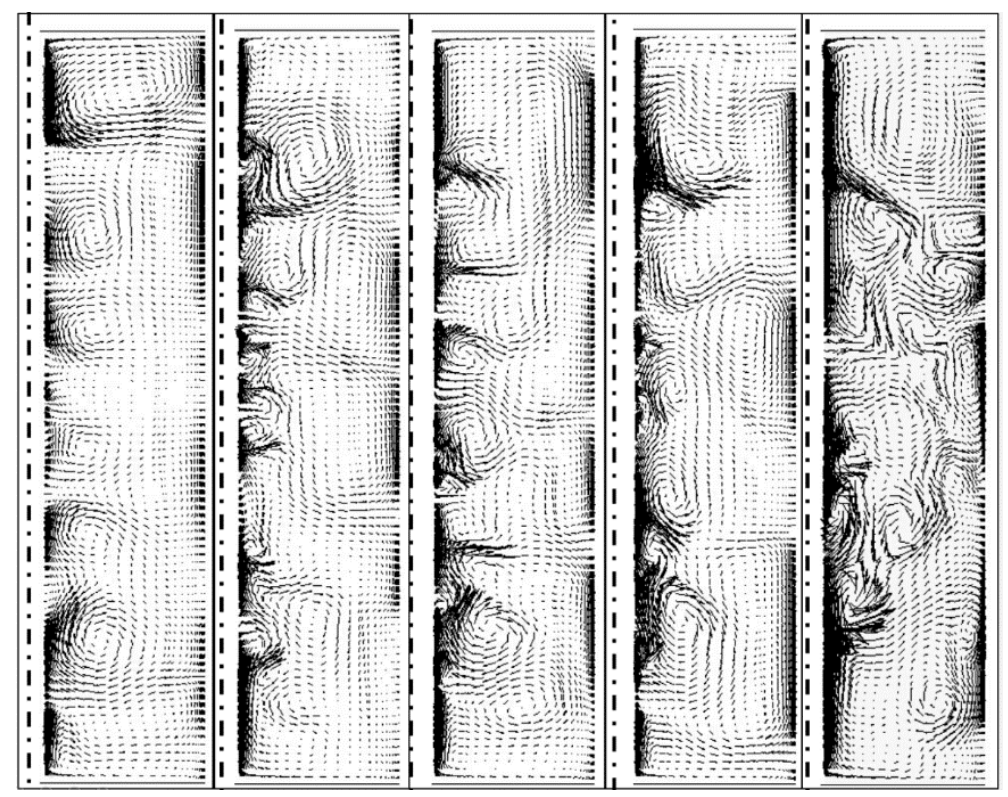

Fig. 5 The velocity fields at $f_{k}$ (from left to right) $f_{k}=1,0.7,0.5,0.3,0.1$

Furthermore, the Nusselt number is also used to compare the prediction of heat transfer, it is calculated with empirical equation and compared to Tachibana's experimental results (Tachibana 
and Fukui, 1964).

$$
N u=\frac{\bar{q} \delta}{k\left(T_{\text {inner }}-T_{\text {outer }}\right)}
$$

where 9 is the average wall heat flux of the outer surface of the stationary wall, $\delta$ is the width of the annular gap, $k$ is the thermal conductivity of water at the average temperature.

Fig. 5 shows the average Nusselt number at different rotational speed. The solid line is the experimental result, and the symbols denote the results of PANS models with different value $f_{k}$, and. Among these PANS models, the $f_{k}=1$ case can be viewed as the standard $k$ - $\varepsilon$ model. The comparison suggests that the $f_{k}=0.3$ case 1 predict a more accurate Nusselt number at $T a \approx 1 \mathrm{E} 12$.

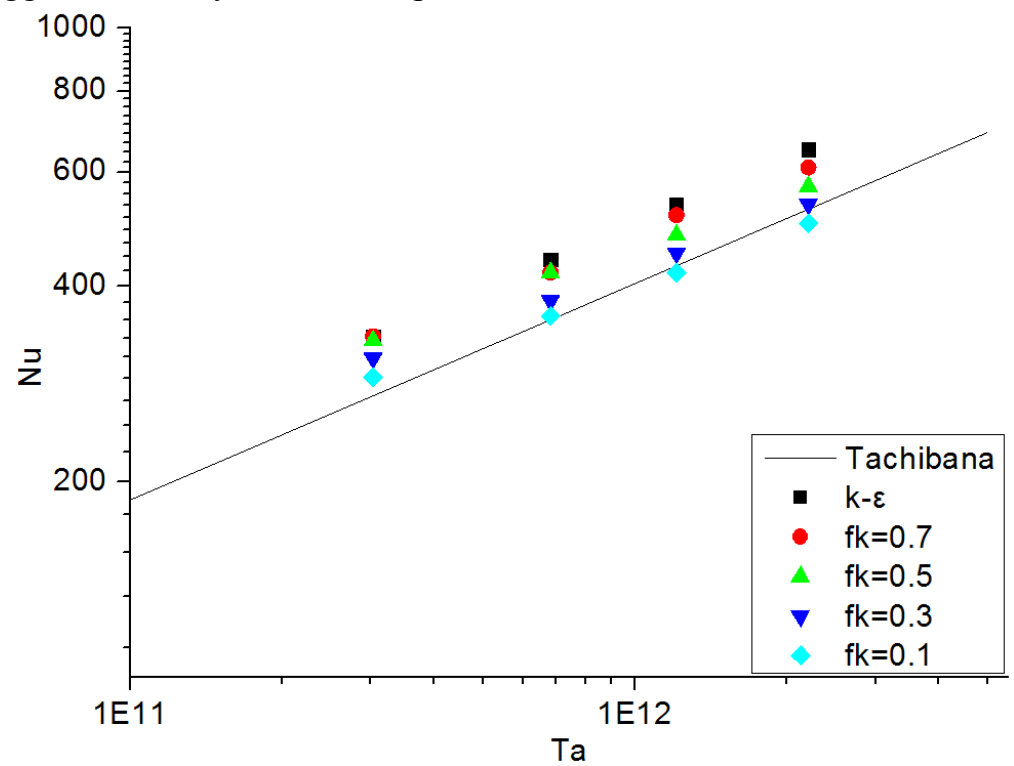

Fig. 6 Comparison of the Nusselt number

\section{Heat transfer in gap flow of flywheel}

\subsection{Computational details}

In this section, the heat transfer model of flywheel is built as conjugate heat transfer, the numerical model is shown in Fig. 7, the top surface of stationary shell is set as constant temperature $566 \mathrm{~K}$, and the bottom surface is $333 \mathrm{~K}$, the hoop surface is adiabatic wall, the surface of flywheel is defined as rotating wall $(1780 \mathrm{rpm})$, the inlet velocity is set as mass flow rate at constant temperature, the outlet is set as opening, and other interfaces between solid domain and fluid domain are treated as conservative interface flux. In addition, the temperature distribution in the axial direction is from $333 \mathrm{~K}$ to $566 \mathrm{~K}$, so the variation material property of the water is used the heat transfer simulation.

The computational model is shown in Fig. 7, the number of grid in fluid domain is 2.67E6, and for capturing the smaller scale turbulent motions in annular region, the finer grid distribution is used for the annular gap flow, the grid dimension is $40 \times 250$ (radial $\times$ length). Besides that, the grid resolution of solid is coarser than that in the fluid domain. 


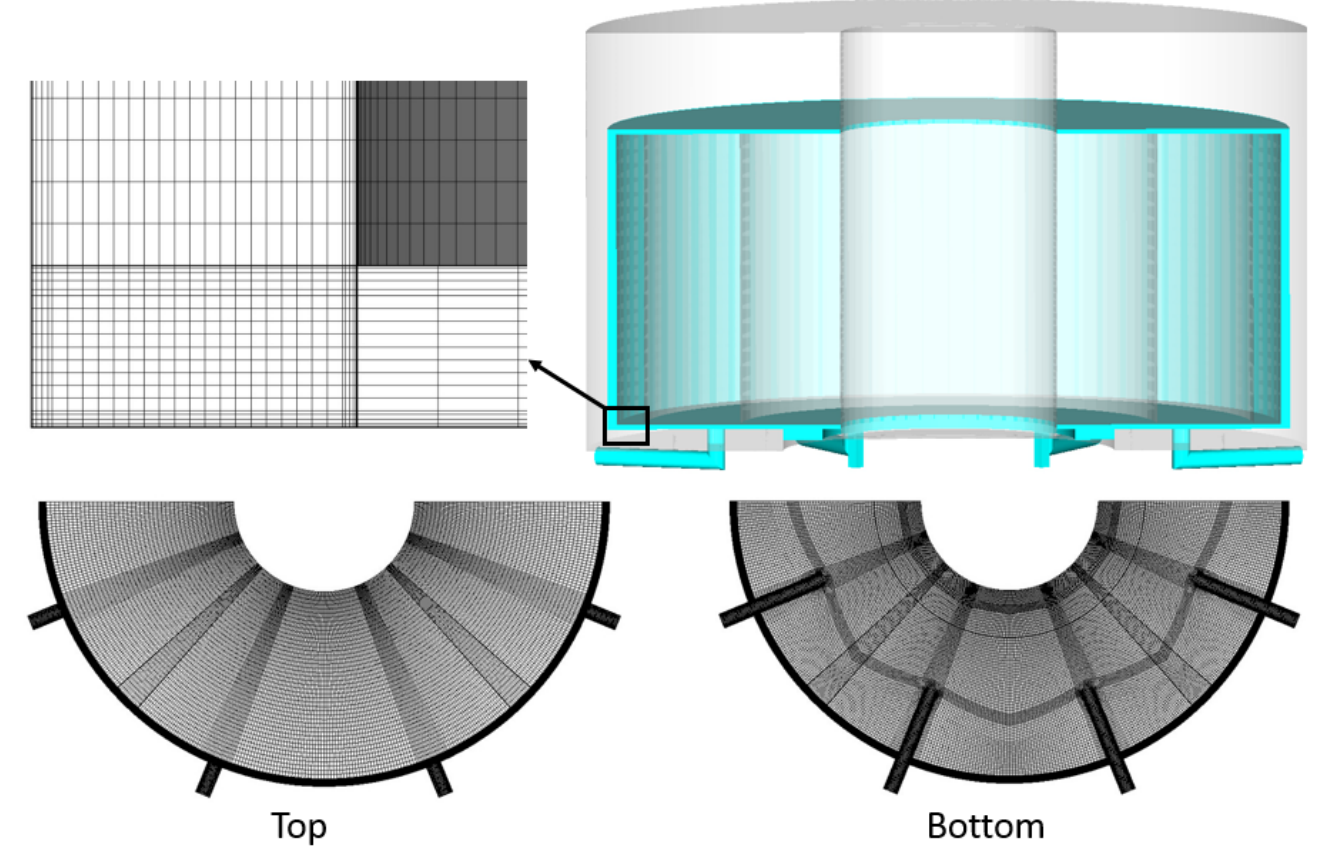

Fig. 7 Conjugate heat transfer model of flywheel

\subsection{Flow structure of the annular gap flow}

Fig. 8 shows that the flow state has eight Taylor vortices (Seven pairs), on the annular surface of the flywheel, the letter 'I' stands for 'Inflow' boundary of a pair of Taylor vortices, the flow is from the stationary surface to the annular surface of flywheel. Conversely, 'O' stands for 'Outflow' boundary of a pair of Taylor vortices, the flow is from the annular surface to the stationary surface. Compared with the $k-\varepsilon$ simulation, the PANS model with $f_{k}=0.3$ can successfully predict smaller scale turbulent motions vortices arise between a pair of Taylor vortices.
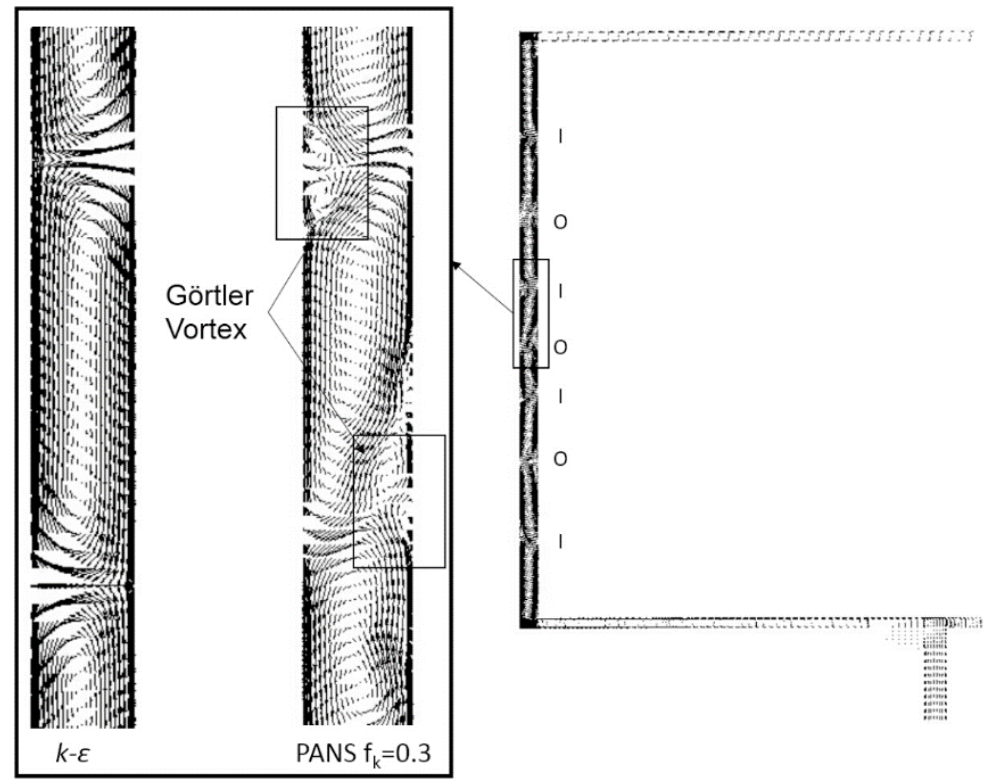

Fig. 8 Velocity field in the annular gap flow of flywheel

Fig. 9 shows that the formation of near-wall organized pattern with $k-\varepsilon$ and PANS model. The 'Band' pattern is demonstrated by the wall shear stress contour of $k-\varepsilon$, which indicates the presence of large scale Taylor vortices. On the contrary, the 'Herringbone' streaks are observed 
with PANS simulation. According to the most author's investigations, the 'Herringbone' streak consist of smaller scale turbulent motions called Görtler vortices (Barcilon et al., 1979; Dong, 2008b). Görtler vortices have the same characteristics as Taylor vortices consist of a pair of contrarotation vortices, but Görtler vortices have some unique characteristics. Firstly, Görtler vortices are only observed at sufficient high Renolds number, and vanish from the wall as the decreasing of Renolds number. Secondly, Görtler vortices originate at the boundaries of Taylor vortices near the wall. Thirdly, Görtler vortices distribution is random 'Band' region at the boundary of Taylor vortices.

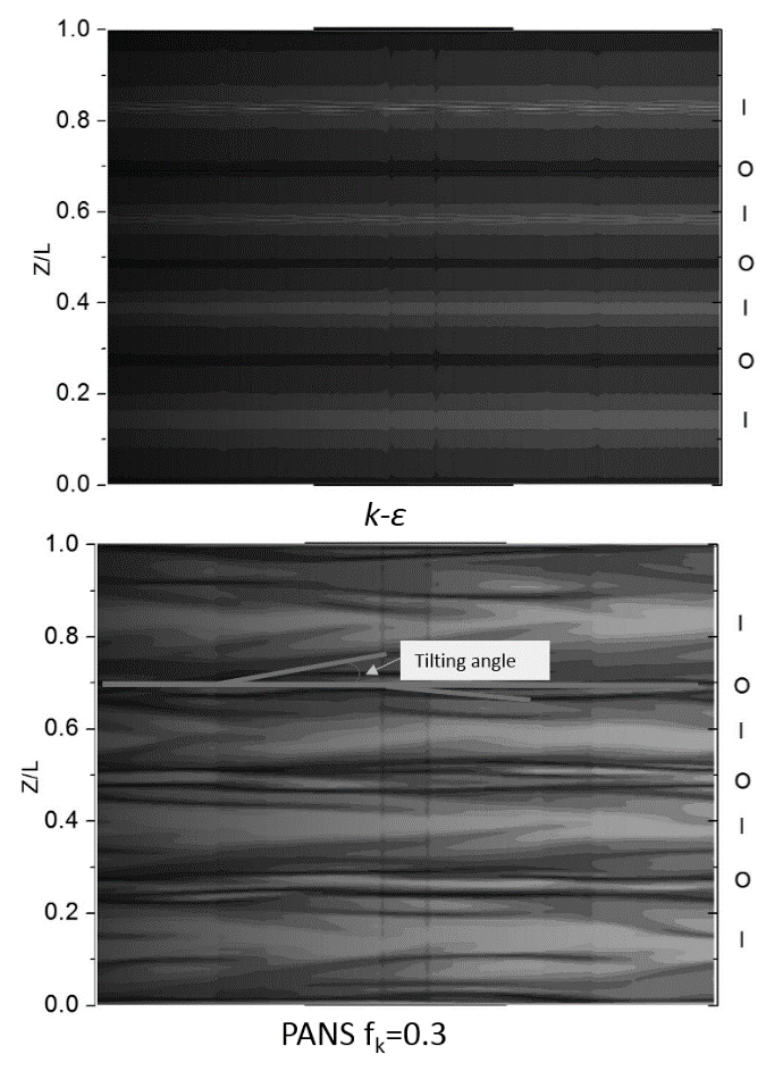

Fig. 9 Shear stress distribution in the annular gap flow

\subsection{Temperature distribution of the annular gap flow in axial direction}

Fig. 10 shows the temperature axial distribution along the surface of the flywheel, the temperature gradient at 'Outflow' is greater than that at the 'Inflow'. According to Fénot's review, the hot and cold flow mix at the 'Outflow' boundary of Taylor vortices, so the temperature gradient is greater than the other locations. In addition, the temperature gradient at Taylor vortices PANS predicted is larger than $k-\varepsilon$, and the maximum temperature is greater than the mixing temperature, and the minimum temperature is lower than the mixing temperature. The reason for the interesting phenomenon can be deduced as the Görtler vortices form a second temperature mixing region at the boundaries of Görtler vortices. 


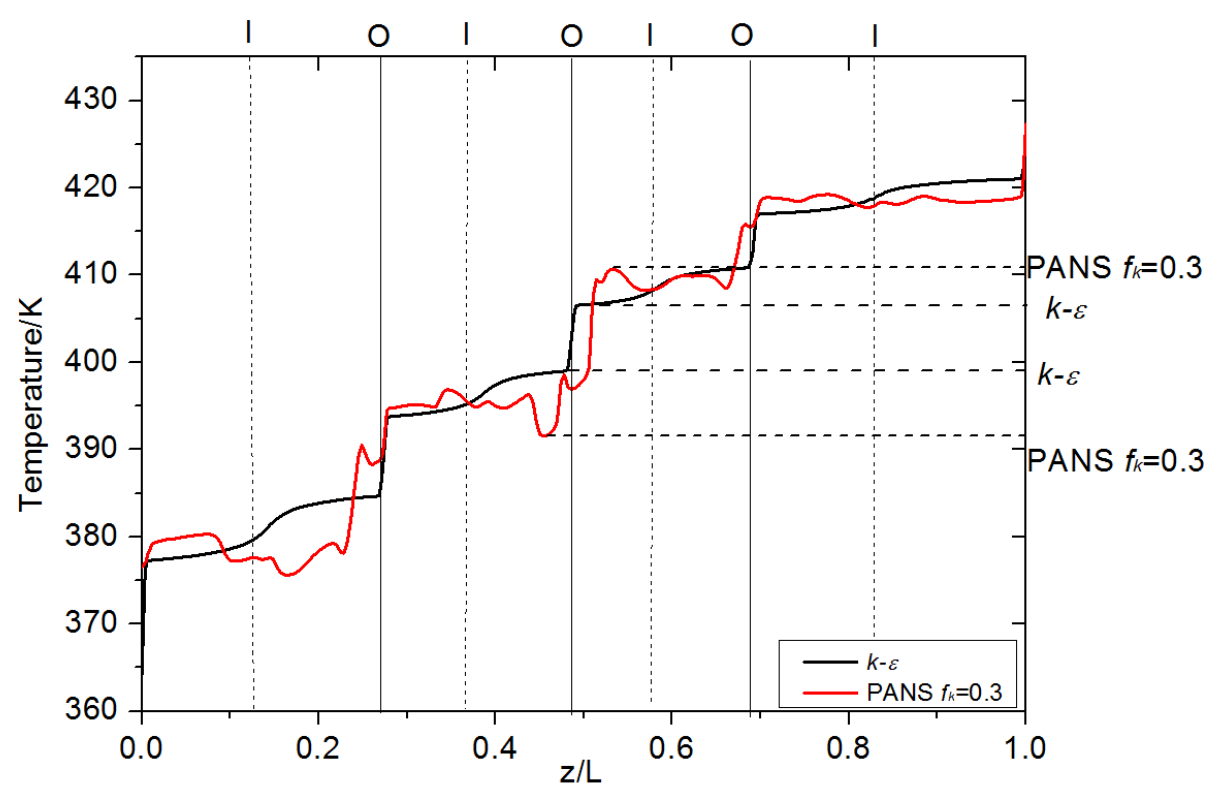

Fig. 10 Temperature distribution of the annular surface of the flywheel in axial direction

This deduction might receive the support of Dong's research and Chouippe's bubble dispersion research. Dong's simulation illustrates that the Görtler vortices are not parallel to the boundary of Taylor vortices, the stretched Görtler vortices extend away from the wall to the core of the Taylor vortices (Dong, 2008b). Furthermore, the numerical simulation Chouippe conducted suggests that the bubbles are attracted to the inner cylinder and cumulated near the inner cylinder in 'Outflow' regions due to the stretched Görtler vortices (Chouippe et al., 2014). In short, the Görtler vortices have the same characteristic as pump, it can accumulate and transport the water from the other regions.

Based on the PANS simulation, the stretched Görtler vortices not only influence the flow state of the gap flow of flywheel, but also play a key role in the heat transfer. The local Nusselt number is chosen to analyze the effect of the Görtler vortices, it is also calculated with Eq. (6Error! Reference source not found., where the parameters of this equation are local value rather than the average value, the Nussel number distribution along the axis of the stationary surface is plotted in Fig. 11. It's worth noting that the maximum Nusselt number is associated with the location of Görtler vortices at the location of 'Outflow' boundaries, and the Nusselt number distribution is anti-symmetry. Obviously, it is evident that the results of PANS model is different with $k-\varepsilon$, because the $k-\varepsilon$ cannot predict the presence of Görtler vortices, so the local heat transfer is not more accurate. 


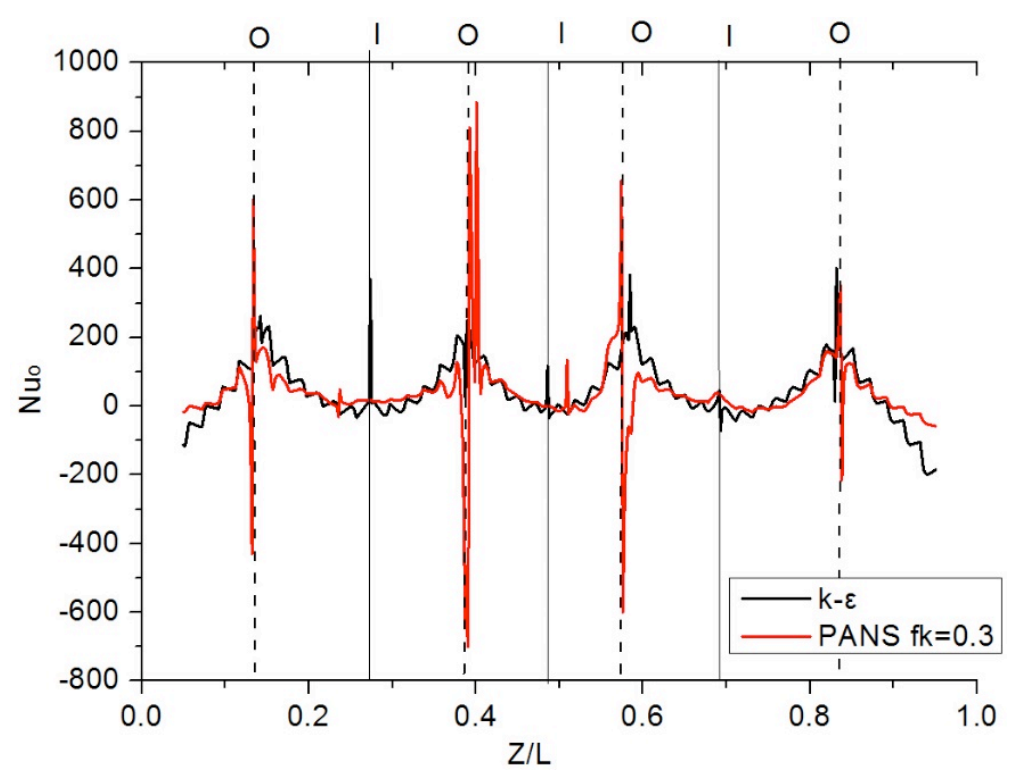

Fig. 11 Nusselt number distribution along the axis of the stationary surface

\subsection{Temperature distribution of the annular gap flow in radial direction}

For analyzing the effect of Görtler vortices on the temperature distribution along the radial of annular gap flow, three paths are selected at the boundary of Taylor vortices to analyze the temperature mixing process, the three path along the boundary of a pair Taylor vortices, which are plotted in Fig. 12, and the three path are denoted as 'Top', 'Mid' and 'Bot'.
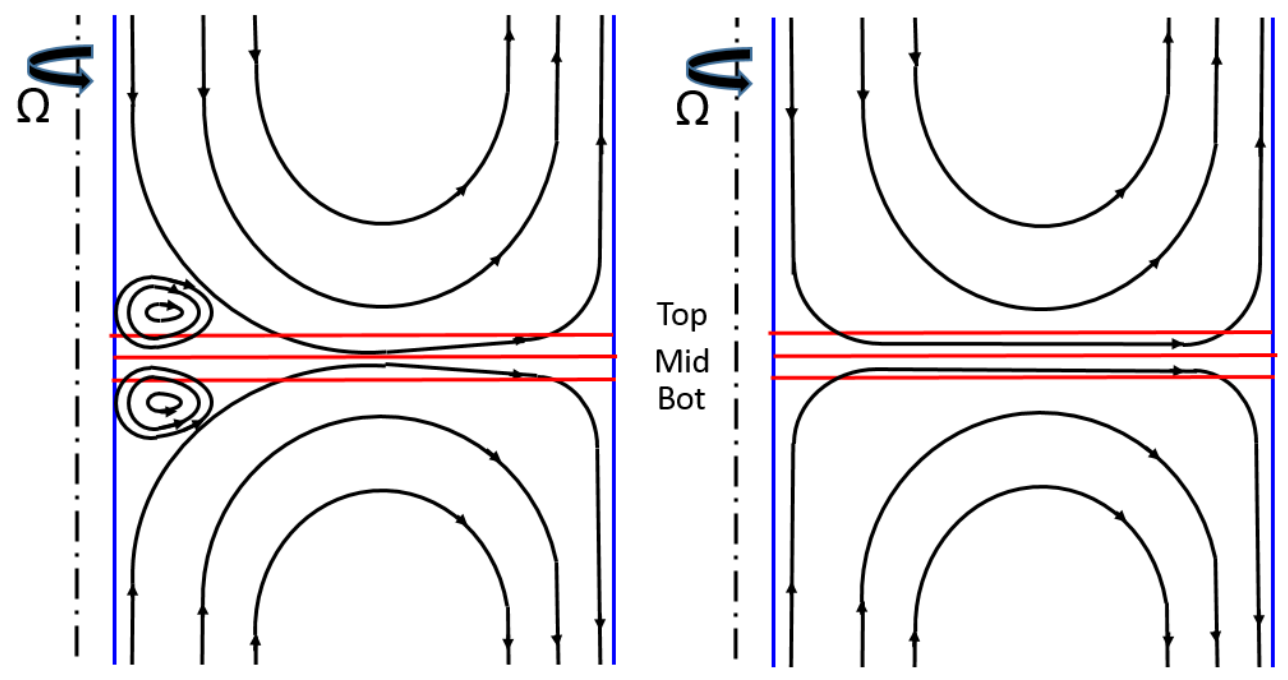

Fig. 12 Three paths definition at the boundary of a pair of Taylor vortices

In Fig. 13, the dimensionless temperature distributions along three paths are depicted in the same figure to reveal the effect of Görtler vortices. The solid symbol line is the result of $k-\varepsilon$ simulation, and the void symbol line is PANS simulation. The short dash line is the vertical velocity distribution along the middle line. When the vertical velocity equals to $0 \mathrm{~m} / \mathrm{s}$, which suggests this location is the boundary of Taylor vortex and Görtler vortex. Obviously, PANS and $k-\varepsilon$ have the different temperature distribution of three paths, the Görtler vortex change the temperature distribution at the annular surface to flywheel, furthermore, the hot water and cold water are mixed by the Görtler vortex.

Fig. 14 illustrates the temperature mixing processes in the annular gap. Temperature mixing processes consist of two independent processes at the same time, one is the temperature mixing of 
Taylor vortices, and another one is the temperature mixing of Görtler vortices. After two temperature mixing processes of cold and hot water, the temperature difference near the wall are minished. In addition, the Görtler vortices always have the opposite rotation direction to Taylor vortices, so the start point of temperature mixing process occurs at the center of annular gap. On the contrary, in $k-\varepsilon$ simulation only present the temperature mixing process of Taylor vortices, so the temperature difference at the outflow of Taylor vortices, i.e. the starting point of the temperature mixing process.

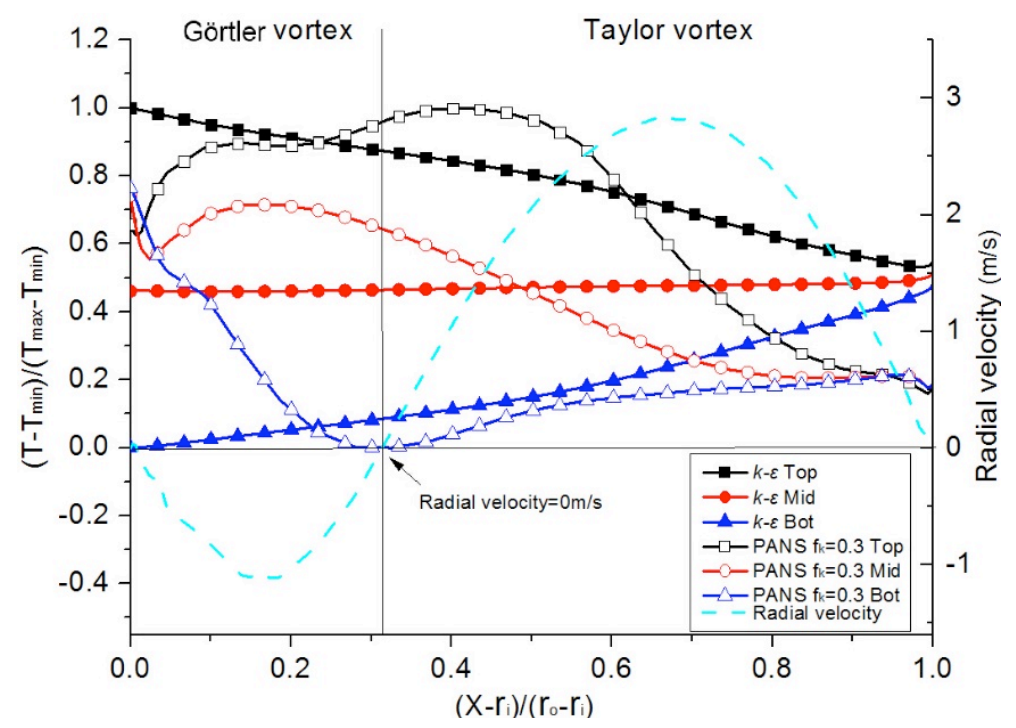

Fig. 13 Temperature distribution at boundary of Taylor vortices in annular gap

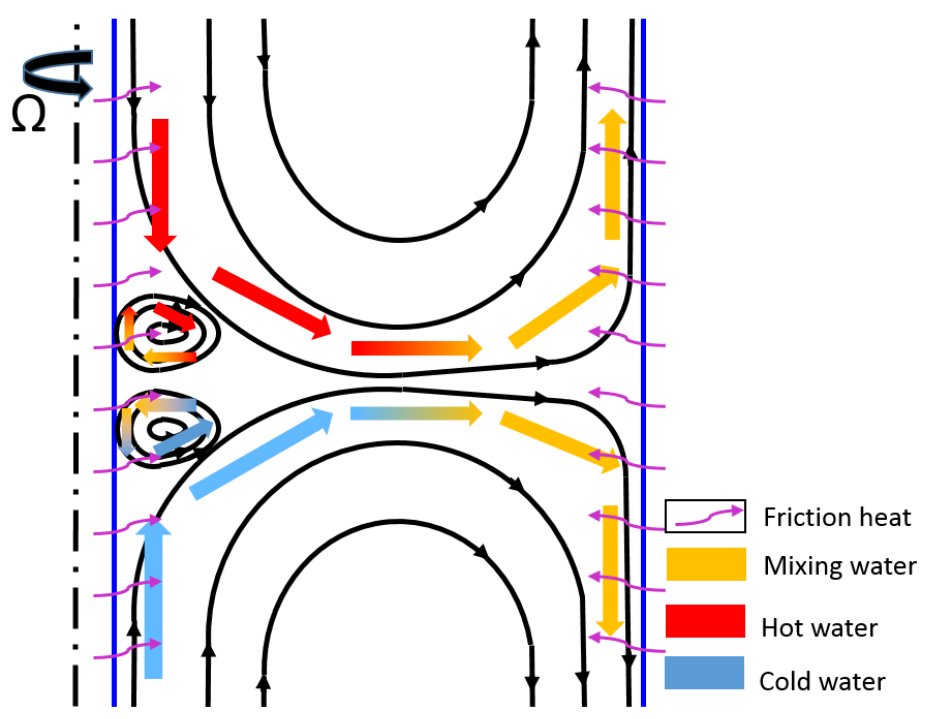

Fig. 14 Heat transfer in the annular gap flow of flywheel

\section{Conclusion}

In this paper, a numerical analysis is conducted for analyzing the effect of the small turbulent motions on the heat transfer in the annular gap flow of RCP flywheel. Based on the previous researches, when the Taylor number reaches up to 1E12, a secondary flow occurs at the boundary of Taylor vortices, which is also called Görtler vortices. Although the DNS can capture the small scale turbulent motions, it will cost a great computational resources, and RANS is hard to capture the small turbulent motions due to the overestimated eddy viscosity. Therefore, a hybrid method 
of DNS and RANS called PANS method is used to analyze the effect of the Görtler vortices on heat transfer.

By comparing with Tachibana's experiment, the validity of the PANS model is confirmed. In addition, the PANS model with $f_{k}=0.3$ present a more accurate Nusselt number than $k-\varepsilon$, which is also used to analyze the heat transfer of the annular gap flow of flywheel. At the boundaries of the Taylor vortices, the stretched Görtler vortices carry the colder or hotter water from the core of Taylor vortices to the boundaries region, so the local temperature gradient in axial direction is larger than that of $k-\varepsilon$. Furthermore, at the boundary of Görtler vortices, another temperature mixing process is found, which influences the temperature distribution in the radial direction, the heat transfer processes in the annular gap flow of flywheel is illustrated in Fig. 14.

\section{Acknowledgments}

This work in this paper is supported by National Science Foundation of China (No.51406114).

\section{References}

Andereck, C.D., Liu, S.S., Swinney, H.L., 1986. Flow regimes in a circular Couette system with independently rotating cylinders. J. Fluid Mech 164, 155-183.

Aoki, H., Nohira, H., Arai, H., 1967. Convective heat transfer in an annulus with an inner rotating cylinder. Bulletin of JSME 10, 523-532.

Barcilon, A., Brindley, J., 1984. Organized structures in turbulent Taylor-Couette flow. J Fluid Mech 143, 429-449.

Barcilon, A., Brindley, J., Lessen, M., Mobbs, F., 1979. Marginal instability in Taylor-Couette flows at a very high Taylor number. J Fluid Mech 94, 453-463.

Batten, W., Bressloff, N., Turnock, S., 2002. Numerical simulations of the evolution of Taylor cells from a growing boundary layer on the inner cylinder of a high radius ratio Taylor-Couette system. Phys Rev E 66, 0663021-0663025.

Becker, K., Kaye, J., 1962. The influence of a radial temperature gradient on the instability of fluid flow in an annulus with an inner rotating cylinder. Journal of Heat Transfer 84, 106-110.

Bilson, M., Bremhorst, K., 2007. Direct numerical simulation of turbulent Taylor-Couette flow. J Fluid Mech 579, 227-270.

Brauckmann, H.J., Eckhardt, B., 2013. Direct numerical simulations of local and global torque in Taylor-Couette flow up to $R e=30000$. J Fluid Mech 718, 398-427.

Chouippe, A., Climent, E., Legendre, D., Gabillet, C., 2014. Numerical simulation of bubble dispersion in turbulent Taylor-Couette flow. Phys Fluids 26, 0433041-04330423.

Dong, S., 2008a. Gortler Vortices in Turbulent Taylor-Couette Flow, 46th AIAA Aerospace Sciences Meeting and Exhibit, Reno, Nevada, pp. 1-9.

Dong, S., 2008b. Herringbone streaks in Taylor-Couette turbulence. Phys Rev E 77, 0353011-0353014.

Fénot, M., Bertin, Y., Dorignac, E., Lalizel, G., 2011. A review of heat transfer between concentric rotating cylinders with or without axial flow. Int J Therm Sci 50, 1138-1155.

Fröhlich, J., von Terzi, D., 2008. Hybrid LES/RANS methods for the simulation of turbulent flows. Progress in Aerospace Sciences 44, 349-377.

Girimaji, S.S., 2006. Partially-averaged Navier-Stokes model for turbulence: A Reynolds-averaged Navier-Stokes to direct numerical simulation bridging method. Journal of Applied Mechanics 73, 413-421.

Girimaji, S.S., Abdol-Hamid, K.S., 2005. Partially Averaged Navier-Stokes Model for Turbulence: 
Implementation and Validation, AIAA paper, Reno, Nevada, pp. 1-14.

Girimaji, S.S., Srinivasan, R., Jeong, E., 2003. PANS turbulence model for seamless transition between RANS and LES: fixed-point analysis and preliminary results, ASME/JSME 2003 4th Joint Fluids Summer Engineering Conference. American Society of Mechanical Engineers, pp. 1901-1909.

Lakshmipathy, S., Girimaji, S.S., 2010. Partially averaged Navier-Stokes (PANS) method for turbulence simulations: flow past a circular cylinder. Journal of Fluids Engineering 132, 121202-121201-121202-121209.

Mallock, A., 1896. Experiments on fluid viscosity. Philosophical Transactions of the Royal Society of London. Series A, Containing Papers of a Mathematical or Physical Character 187, 41-56.

Spalart, P.R., Shur, M., 1997. On the sensitization of turbulence models to rotation and curvature. Aerosp Sci Technol 1, 297-302.

Tachibana, F., Fukui, S., 1964. Convective heat transfer of the rotational and axial flow between two concentric cylinders. Bulletin of JSME 7, 385-391.

Taylor, G.I., 1923. Stability of a Viscous Liquid Contained between Two Rotating Cylinders. Philosophical Transactions of the Royal Society A: Mathematical, Physical and Engineering Sciences 223, 289-343.

Tzeng, S.-C., 2006. Heat transfer in a small gap between co-axial rotating cylinders. Int Commun Heat Mass 33, 737-743.

van Gils, D.P.M., Huisman, S.G., Bruggert, G.W., Sun, C., Lohse, D., 2011. Torque Scaling in Turbulent Taylor-Couette Flow with Co- and Counterrotating Cylinders. Physical review letters 106, 1-4.

Wei, T., Kline, E., Lee, S.-K., Woodruff, S., 1992. Görtler vortex formation at the inner cylinder in Taylor-Couette flow. J Fluid Mech 245, 47-68. 